\title{
VARIAÇÃO ESTRUTURAL FOLIAR DE ESPÉCIES MEDICINAIS EM CONSÓRCIO COM ERVA-MATE, SOB DIFERENTES INTENSIDADES LUMINOSAS
}

\author{
Maria Regina Torres Boeger ${ }^{1}$, Ari Espíndola Júnior ${ }^{2}$, Agenor Maccari Júnior ${ }^{3}$, \\ Carlos Bruno Reissmann ${ }^{4}$, Ana Cristina Atala Alves ${ }^{5}$, Fernanda Lessa Rickli $^{6}$ \\ ${ }^{1}$ Bióloga, Dra ., Depto. de Botânica, UFPR, Curitiba, PR, Brasil - rboeger@ufpr.br \\ ${ }^{2}$ Biólogo, M.Sc., Curitiba, PR, Brasil - ari_junior_bio@hotmail.com \\ ${ }^{3}$ Eng. Agrônomo, Dr., Depto. de Solos e Engenharia Agrícola, UFPR, Curitiba, PR, Brasil - maccari@ufpr.br \\ ${ }^{4}$ Eng. Florestal, Dr., Depto. de Solos e Engenharia Agrícola, UFPR, Curitiba, PR, Brasil - reissman @ufpr.br \\ ${ }^{5}$ Bióloga, M.Sc., Curitiba, PR, Brasil - anaatala@ig.com.br \\ ${ }^{6}$ Eng $^{\mathrm{a}}$. Agrônoma, UEPG, Ponta Grossa, PR, Brasil - fer_lessa@yahoo.com.br
}

Recebido para publicação: 26/09/2007 - Aceito para publicação: 04/06/2008

\begin{abstract}
Resumo
O objetivo deste estudo foi analisar a estrutura foliar de quatro espécies medicinais (Bauhinia forficata, Maytenus ilicifolia, Mikania glomerata e Vitex megapotamica) consorciadas com Ilex paraguariensis (erva-mate), em três condições de luz, para fornecer subsídios do melhor regime de luz para o cultivo dessas espécies. Na fazenda Capão Bonito, município de Castro, PR, o experimento foi realizado com três tratamentos de luz: 100\% (pleno sol), 26,23\% (meia-sombra) e 13,83\% (sombra) de luminosidade. Comparando-se as características morfológicas entre os três tratamentos e as espécies estudadas, $M$. ilicifolia, $V$. megapotamica e $B$. forficata apresentaram os mesmos padrões de variação, excetuando-se a área foliar em $B$. forficata, a espessura do parênquima paliçádico em $M$. ilicifolia e a concentração de clorofila, que foi diferente entre as três espécies. M. glomerata foi a espécie com maior plasticidade foliar, com exceção da concentração de clorofila. Os valores médios de AEF indicaram que o tratamento sombra é o que oferece maior biomassa por unidade de área, para todas as espécies. Entretanto, estudos adicionais de quantificação de princípio ativo, taxas de crescimento, respostas sazonais e condições nutricionais diferenciadas nos diferentes regimes de luz podem complementar as informações para a adoção de métodos de cultivo mais eficientes dessas espécies medicinais.

Palavras-chave: Morfologia foliar; plantas medicinais; variação fenotípica.
\end{abstract}

\begin{abstract}
Structural variation of leaves of medicinal species associated with "erva-mate", under different light intensities. The objective of this study was to analyze the leaf morphology of four medicinal species (Bauhinia forficata; Maytenus ilicifolia; Mikania glomerata, and Vitex megapotamica) associated with Ilex paraguariensis ("erva-mate"), at three light conditions and to indicate the best light regime for the cultivation of these species. The experiment was set up in the Fazenda Capão Bonito (25 50' 64' 'S and 49 43' 69' 'W) in Castro, PR, Brazil, and was composed by three light treatments: $100 \%$ (full sunlight), 26,23\% (half-shade) and 13,83\% (shade). Comparing the morphological characteristics among treatments and species, M. ilicifolia, V. megapotamica, and B. forficata presented the same variation pattern, except for the leaf area in $B$. forficate, the thickness of the palisade parenchyma in M. ilicifolia, and the chlorophyll concentration which was different among the three species. $M$. glomerata had the highest foliar plasticity, except for the chlorophyll concentration. The mean values of AEF indicate that the shade treatment produces the highest biomass, by unity of area, for all species. However, additional studies on the quantification of active principles, growth rates, seasonal responses, and distinct nutritional conditions among the light treatments can provide additional information for the implementation of more efficient methods of cultivation for these medicinal species.
\end{abstract}

Keywords: Medicinal species; phenotypic variability; leaf morphology. 


\section{INTRODUÇÃO}

A cultura de Ilex paraguariensis A. St.- Hil. (Aquifoliaceae), conhecida popularmente como erva-mate, possui vínculos históricos com o estado do Paraná (Brasil), sendo que na região Sul ela constitui uma das principais fontes de emprego e renda, especialmente para pequenos e médios agricultores (DEDECEK; RODIGHERI, 1999). No entanto, o tempo de espera para cultivares plantados atingirem o porte para colheita é longo, o que torna oneroso para o produtor a manutenção da área de cultivo a curto prazo. Uma alternativa para esse problema é o consórcio de culturas de alto valor agregado com os ervais.

O valor agregado da comercialização de plantas medicinais constitui uma opção vantajosa de obtenção de renda alternativa para o pequeno produtor, a qual, atendendo a demanda do mercado, pode auxiliar na redução do extrativismo e propiciar opções para o controle de qualidade na produção de fármacos (MILANEZE-GUTIERRE et al., 2003). Informações sobre as variações da morfologia foliar dessas espécies em diferentes regimes de luz, além de contribuir para o delineamento de métodos de cultivo, podem auxiliar na avaliação da viabilidade de sua utilização em reflorestamentos de áreas improdutivas de fazendas, fomentando ações de recuperação e recomposição do bosque nativo ou iniciativas de manejo de cultivo. Desse modo, as características morfológicas representam, do ponto de vista produtivo, um fator importante tanto na safra que se pretende obter quanto na qualidade da droga vegetal fabricada a partir da matéria-prima cultivada.

A luz é um dos mais importantes fatores abióticos que afeta a fisiologia e a morfologia dos vegetais. Os ajustes morfofisiológicos que ocorrem nas plantas são relacionados principalmente com a manutenção da eficiência do balanço entre o ganho de carbono, pela fotossíntese, e a perda de água, pela transpiração (GIVNISH, 1988; TAIZ; ZEIGER, 2004). Consequentemente, as folhas desenvolvem características estruturais para otimizar esse processo (VOGELMANN et al., 1996; SMITH et al., 1997), o que reflete na produção de massa verde das plantas.

Os vários estudos da influência da luz sobre o crescimento vegetal têm indicado que plantas de ambientes sombreados geralmente alocam maior quantidade de biomassa nas folhas e possuem maior área foliar por unidade de massa (POORTER, 1999; LEE et al., 2000). Por outro lado, plantas expostas à luz solar intensa investem em biomassa radicial, para compensar a perda de água por transpiração, e, devido às altas taxas fotossintéticas, produzem maior biomassa por unidade de área foliar e altas taxas de renovação das folhas (POORTER, 1999).

Este estudo faz parte de um projeto maior, que envolve a avaliação do crescimento de quatro espécies medicinais - Mikania glomerata Spreng. (Asteraceae); Bauhinia forficata Link. (Fabaceae); Maytenus ilicifolia Mart. ex Reiss. (Celastraceae) e Vitex megapotamica (Spreng.) Moldenke (Lamiaceae) - nativas da Floresta Ombrófila Mista (Floresta com Araucária), consorciadas a Ilex paraguariensis A. St.-Hil. (Aquifoliaceae), sob três condições de luminosidade distintas (pleno sol, meia sombra e sombra), dadas respectivamente por diferentes ambientes (campo, vegetação pouco densa e bosque).

Maytenus ilicifolia (Schrad.) Planch. (Celastraceae), conhecida popularmente como espinheirasanta, cancerosa, cancoroso, cancrosa (CORRÊA JR. et al., 1991), é uma espécie nativa da região Sul do Brasil, natural do sub-bosque da Floresta Ombrófila Mista (LORENZI, 1998). A espinheira-santa é utilizada na medicina popular para o combate de dispepsias e gastralgias hiperclorídricas no estômago, assim como na prevenção de fermentações e formações de gases no intestino (PIO CORRÊA, 1984).

Vitex megapotamica (Spreng.) Moldenke (Lamiaceae), conhecida vulgarmente por tarumã, tarumã-preta, tarumã-de-montevidéu, é uma espécie arbórea, nativa da região Sul do Brasil, predominante dos sub-bosques da Floresta Ombrófila Mista (LORENZI, 1998). Seus folíolos coriáceos podem atingir de 5 a $9 \mathrm{~cm}$ de comprimento e possuem nervuras bastante salientes na face abaxial (PIO CORRÊA, 1984). As folhas, em infusão, são usadas no combate ao colesterol, como diurético e depurativo do sangue (LORENZI, 1998).

Bauhinia forficata Link. (Fabaceae), conhecida como pata-de-boi, pata-de-vaca, unha-de-vaca e morró (PIO CORRÊA, 1984), é uma planta espinhenta, variando de 5 a $9 \mathrm{~m}$ de altura, com tronco tortuoso de 30 a $40 \mathrm{~cm}$ de diâmetro, folhas glabras ou levemente pubescentes na face dorsal, com lâmina profundamente dividida, de 8 a $12 \mathrm{~cm}$ de comprimento (LORENZI, 1998). As folhas, caules e raízes das espécies de Bauhinia são amplamente utilizadas na forma de chás e outras preparações fitoterápicas para o tratamento de algumas infecções urinárias e para regulação da glicemia (SILVA; CECHINEL FILHO, 2002). 
Mikania glomerata Spreng. (Asteraceae), conhecida como guaco, é um subarbusto de hábito volúvel, com uso medicinal amplamente difundido na região Sul do Brasil, ocorrendo em beira e interior de mata (RITTER; WAECHTER, 2004). Apresenta ramos lenhosos, cilíndricos, com folhas pecioladas, cordiformes, ápices agudos e base cordiforme (PIO CORRÊA, 1984). As folhas apresentam atividade anti-inflamatória, efeito inibitório da musculatura intestinal e uterina e atividade broncodilatadora devido à presença das cumarinas (CELEGHINI et al., 2001).

Neste trabalho, são apresentados os resultados comparativos referentes ao estudo morfológico dessas espécies, com o objetivo de contribuir para o delineamento de métodos de cultivo e auxiliar na avaliação do uso dessas espécies em reflorestamento de áreas improdutivas de fazendas, fomentando ações de recuperação e recomposição do sub-bosque nativo.

\section{MATERIAL E MÉTODOS}

O experimento foi instalado na Fazenda Capão Bonito (2550'64' 'S, 4943'69' W), município de Castro, PR, no Primeiro Planalto Paranaense. A região apresenta temperatura média anual de $16,3^{\circ} \mathrm{C}$, com médias do mês mais quente de $23,7^{\circ} \mathrm{C}$ e do mês mais frio de $11,7^{\circ} \mathrm{C}$. A precipitação anual é de $1.469,1 \mathrm{~mm}$, sendo janeiro o mês mais chuvoso e julho o mês mais seco. A região é climaticamente caracterizada como $C f b$ (clima quente-temperado, sempre úmido), de acordo com a classificação de Koeppen (MAACK, 1981).

As três condições de intensidade luminosa foram obtidas através do manejo da cobertura vegetal natural do local: tratamento pleno sol, em que predomina vegetação herbácea, com intensidade luminosa de 100\%; tratamento meia sombra, que corresponde à área de mata, com intensidade luminosa de 26,23\%; e tratamento sombra, que corresponde ao sub-bosque, com intensidade luminosa de $13,83 \%$. Cada tratamento foi composto por três parcelas, com nove mudas de cada espécie. A intensidade luminosa foi medida em cada um dos tratamentos, com luxímetro digital, para aferir o real sombreamento sobre as plantas. As medidas em campo foram feitas em três horários: 9:00, 12:00 e 15:00 h, em três dias consecutivos, em intervalos de três meses a cada série de medições. Através dessas medições, foi possível quantificar a luminosidade incidente em cada tratamento. O solo da área é homogêneo e foi classificado como Cambissolo Háplico Distrófico, com alta relação silte-argila, podendo ser considerado em processo de formação e liberação de nutrientes a partir da intemperização do silte (REISSMANN, dados não publicados).

De cada espécie e para cada tratamento, foram coletadas 34 folhas totalmente expandidas, entre os $3^{\circ}$ e $4^{\circ}$ nós (sentido ápice-base dos ramos). Considerando-se que Vitex megapotamica apresenta folha composta digitada, utilizou-se o folíolo mediano (BONGERS; POPMA, 1990). No tratamento meiasombra, a intensa herbivoria danificou várias mudas de $B$. forficata, limitando o número de mudas sadias e inviabilizando a coleta dessa espécie nesse tratamento. As folhas coletadas para o estudo morfológico e anatômico foram acondicionadas em sacos plásticos previamente umedecidos e transportadas para o laboratório.

A massa fresca $(\mathrm{g})$ e a massa seca $(\mathrm{g})$ foram estimadas em balança analítica de precisão Celtac. Para a massa seca, as folhas e folíolos foram prensados entre folhas de jornal e secos em estufa à temperatura de $65{ }^{\circ} \mathrm{C}$, até atingirem peso constante. O conteúdo de água $(\mathrm{g})$ foi estimado a partir da diferença entre a massa fresca e a massa seca. Posteriormente, a área foliar $\left(\mathrm{cm}^{2}\right)$ foi calculada nas folhas previamente secas a partir da imagem digitalizada em scanner de mesa, acoplado ao computador, pelo programa SIGMASCAN-PRO (versão 5.0, SPSS Inc., Chicago, USA, 1995). A área específica foliar $\left(\mathrm{AEF}, \mathrm{cm}^{2} \cdot \mathrm{g}^{-1}\right)$ foi estimada a partir da razão $\mathrm{AEF}=\mathrm{AF} / \mathrm{MS}$, em que $\mathrm{AF}=$ área foliar e MS = massa seca (WITKOWSKI; LAMONT, 1991). As densidades estomática e de tricomas $\left(\mathrm{n}^{\circ} . \mathrm{mm}^{-2}\right)$ foram estimadas através da modelagem da epiderme na região mediana da folha com esmalte de unha sintético incolor e contagem do número de estômatos e tricomas numa área previamente conhecida em microscópio óptico com câmara clara acoplada. Estômatos e tricomas foram contados em dois campos por folha.

Secções transversais semipermanentes da região mediana das folhas foram obtidas com lâmina de barbear, clarificadas com hipoclorito de sódio $15 \%$ e coradas com azul de toluidina a 0,05\% (FEDER; O'BRIEN, 1968). As mensurações das espessuras $(\mu \mathrm{m})$ da lâmina foliar e dos tecidos foram realizadas em microscópio óptico com ocular micrometrada acoplada. 
Para cada tratamento e espécie, seis discos foliares de $1 \mathrm{~cm}^{2}$ de diâmetro foram seccionados e utilizados para a quantificação dos pigmentos foliares (clorofila $a$, clorofila $b$, razão clorofila $a / b$ e clorofila total). A extração foi efetuada com acetona $80 \%$, com centrifugação do extrato em $2500 \mathrm{rpm}$ e leitura de absorbância em espectrofotômetro Hitachi modelo U-2001 (Hitachi, Inc., São Francisco, Ca, USA), sendo aplicados os coeficientes de extinção definidos por Porra et al. (1989).

Os valores médios e respectivos desvios padrões de todas as variáveis quantitativas foram calculados. A comparação dos valores médios entre os três tratamentos de luz foi realizada pela Análise de Variância (ANOVA), utilizando-se o Programa Statistica (versão 6.0 StatSoft, 1nc., Tulsa, USA, 2001). Para cada variável, verificou-se a diferença significativa entre os tratamentos de luz, utilizando-se o teste de comparação múltipla LSD (menor diferença significativa) de Fisher, com 5\% de nível de significância. Utilizou-se o teste de Bartlet para verificar a homogeneidade das amostras, e para as variáveis que desviaram significativamente de uma distribuição normal foi realizado o procedimento de transformação de médias.

\section{RESULTADOS}

A análise de variância (ANOVA) por espécie, entre os tratamentos, mostrou que as espécies medicinais apresentaram padrões diferenciados quanto às características morfológicas investigadas. Os valores médios de área foliar, massa fresca e seca e conteúdo de água não foram diferentes entre os tratamentos para $M$. ilicifolia e $V$. megapotamica. Para $M$. glomerata e $B$. forficata, os valores médios de área foliar foram maiores no tratamento sombra. Somente $M$. glomerata apresentou os maiores valores médios e estatisticamente significantes de massa fresca e seca e conteúdo de água no tratamento sombra (Tabela 1).

Tabela 1. Valores médios e respectivos desvios padrões (entre parênteses) dos atributos morfológicos $(n=20)$ de quatro espécies medicinais sob três condições de luminosidade. Letras diferentes para a mesma variável (mesma coluna), por espécie, significam que os valores são estatisticamente significantes (Fisher, $\mathrm{p}<0,05)$. AEF = área específica foliar.

Table 1. Mean values and respective standard deviation (between parenthesis) of morphological attributes $n=20$ ), of four medicinal species under three light conditions. Different letters for the same variable (same column), per species, mean that the values are statistically significant (Fisher, $\mathrm{p}<0,05)$. AEF $=$ specific leaf area.

\begin{tabular}{|c|c|c|c|c|c|c|}
\hline Espécies & Tratamentos & $\begin{array}{c}\text { Área foliar } \\
\left(\mathrm{cm}^{2}\right)\end{array}$ & $\begin{array}{c}\text { Massa } \\
\text { fresca }(g)\end{array}$ & $\begin{array}{c}\text { Massa } \\
\text { seca (g) }\end{array}$ & $\begin{array}{l}\text { Conteúdo } \\
\text { água (g) }\end{array}$ & $\begin{array}{c}\text { AEF } \\
\left(\mathrm{cm}^{2} \cdot \mathbf{g}^{-1}\right)\end{array}$ \\
\hline \multirow{6}{*}{$\begin{array}{l}\text { Maytenus } \\
\text { ilicifolia }\end{array}$} & \multirow[t]{2}{*}{ Pleno sol } & 7,10 & 0,24 & 0,11 & 0,13 & 63,48 \\
\hline & & $(2,46) \mathrm{a}$ & $(0,08) \mathrm{a}$ & $(0,03) \mathrm{a}$ & $(0,04) \mathrm{a}$ & $(12,13) b$ \\
\hline & \multirow{2}{*}{ Meia-sombra } & 8,20 & 0,28 & 0,13 & 0,15 & 61,91 \\
\hline & & $(3,45) \mathrm{a}$ & $(0,11) \mathrm{a}$ & $(0,04) \mathrm{a}$ & $(0,06) \mathrm{a}$ & $(11,12) b$ \\
\hline & \multirow{2}{*}{ Sombra } & 9,04 & 0,27 & 0,12 & 0,14 & 75,5 \\
\hline & & $(2,67) \mathrm{a}$ & $(0,08) \mathrm{a}$ & $(0,04) \mathrm{a}$ & $(0,04) \mathrm{a}$ & $(5,38) \mathrm{a}$ \\
\hline \multirow{6}{*}{$\begin{array}{l}\text { Vitex } \\
\text { megapotamica }\end{array}$} & \multirow{2}{*}{ Pleno sol } & 19,33 & 0,52 & 0,20 & 0,32 & 103,46 \\
\hline & & $(10,81) \mathrm{a}$ & $(0,27) \mathrm{a}$ & $(0,13) \mathrm{a}$ & $(0,17) \mathrm{a}$ & $(18,26) \mathrm{b}$ \\
\hline & \multirow[t]{2}{*}{ Meia-sombra } & 20,81 & 0,43 & 0,17 & 0,26 & 121,89 \\
\hline & & $(4,39) \mathrm{a}$ & $(0,10) \mathrm{a}$ & $(0,04) \mathrm{a}$ & $(0,06) \mathrm{a}$ & $(21,34) b$ \\
\hline & \multirow[t]{2}{*}{ Sombra } & 24,10 & 0,42 & 0,12 & 0,30 & 185,64 \\
\hline & & $(10,50) \mathrm{a}$ & $(0,18) \mathrm{a}$ & $(0,04) \mathrm{a}$ & $(0,14) \mathrm{a}$ & $(31,40) \mathrm{a}$ \\
\hline \multirow{6}{*}{$\begin{array}{l}\text { Mikania } \\
\text { glomerata }\end{array}$} & \multirow[t]{2}{*}{ Pleno sol } & 39,4 & 1,8 & 0,27 & 1,56 & 39,4 \\
\hline & & $(11,3) \mathrm{c}$ & $(0,5) \mathrm{c}$ & $(0,07) \mathrm{b}$ & $(0,47) \mathrm{c}$ & $(11,3) \mathrm{c}$ \\
\hline & \multirow{2}{*}{ Meia-sombra } & 50 & 2,2 & 0,29 & 1,93 & 50 \\
\hline & & $(9,8) \mathrm{b}$ & $(0,4) \mathrm{b}$ & $(0,08) \mathrm{ab}$ & $(0,36) \mathrm{b}$ & $(9,8) b$ \\
\hline & \multirow{2}{*}{ Sombra } & 91,3 & 4,1 & 0,47 & 3,65 & 91,3 \\
\hline & & $(21,4) \mathrm{a}$ & $(1,2) \mathrm{a}$ & $(0,13) \mathrm{a}$ & $(1,08) \mathrm{a}$ & $(21,4) \mathrm{a}$ \\
\hline \multirow{4}{*}{$\begin{array}{l}\text { Bauhinia } \\
\text { forficata }\end{array}$} & \multirow[t]{2}{*}{ Pleno sol } & 39,9 & 0,83 & 0,33 & 0,51 & 126,3 \\
\hline & & $(15,2) b$ & $(0,40) \mathrm{a}$ & $(0,15) \mathrm{a}$ & $(0,25) \mathrm{a}$ & $(15,7) b$ \\
\hline & \multirow[t]{2}{*}{ Sombra } & 58,8 & 0,89 & 0,31 & 0,57 & 192,1 \\
\hline & & $(16,3) \mathrm{a}$ & $(0,22) \mathrm{a}$ & $(0,07) \mathrm{a}$ & $(0,15) \mathrm{a}$ & $(49,0) \mathrm{a}$ \\
\hline
\end{tabular}


As quatro espécies apresentaram o mesmo comportamento para apenas duas características. No tratamento sombra, os valores médios de AEF foram estatisticamente diferentes e maiores, enquanto que a densidade estomática média foi menor, no tratamento sombra, em relação aos demais tratamentos (Tabelas 1 e 2).

$\mathrm{Na}$ única espécie com folhas anfiestomáticas, B. forficata, a densidade de estômatos da face adaxial variou entre os tratamentos, sendo que, no tratamento pleno sol, as folhas apresentaram os maiores valores significativos de densidade de estômatos (Tabela 2). Mikania glomerata foi a única espécie com tricomas em ambas as faces da folha. A densidade de tricomas da face adaxial dessa espécie foi significantemente maior no tratamento pleno sol. Tricomas na face abaxial estão presentes em $V$. megapotamica, $M$. glomerata e B. forficata, sendo que a densidade média de tricomas foi estatisticamente maior no tratamento pleno sol apenas em V. megapotamica e M. glomerata (Tabela 2).

A espessura total média da lâmina foi maior no tratamento pleno sol apenas em $B$. forficata. Para M. ilicifolia e V. megapotamica, os valores médios foram diferentes e menores no tratamento sombra. Em M. glomerata, a espessura total média foi diferente somente entre os tratamentos sombra e pleno sol, sendo que a maior espessura ocorreu nas folhas do tratamento pleno sol. Com exceção de $M$ ilicifolia, a espessura média do parênquima paliçádico foi diferente entre sombra e as outras condições (meia-sombra e pleno sol) para todas as espécies. A espessura média do parênquima paliçádico no tratamento sombra apresentou os menores valores. Já a espessura média do parênquima esponjoso variou significativamente entre os tratamentos pleno sol e sombra, somente em M. glomerata. B. forficata possui apenas parênquima paliçádico, caracterizando o mesofilo como homogêneo. A razão parênquima paliçádico/esponjoso <1 ocorreu em todos os tratamentos para M. ilicifolia e M. glomerata. Em V. megapotamica, a relação foi $>1$ nos tratamentos pleno sol e meia-sombra (Tabela 3).

Tabela 2. Valores médios e respectivos desvios padrões (entre parênteses) das densidades estomáticas e de tricomas $(n=20)$ de quatro espécies medicinais sob três condições de luminosidade. Letras diferentes para a mesma variável (mesma coluna), por espécie, significam que os valores são estatisticamente significantes (Fisher, $\mathrm{p}<0,05$ ). Legenda: $\mathrm{nc}=$ não-calculado; DEAd $=$ densidade estomática da face adaxial; DEAb = densidade estomática da face abaxial; DTAd = densidade de tricomas da face adaxial; DTAb = densidade de tricomas da face abaxial.

Table 2. Mean values and respective standard deviation (between parenthesis) of stomata and trichomes densities $(n=20)$, of four medicinal species under three light conditions. Different letters for the same variable (same column), per species, mean that the values are statistically significant (Fisher, $\mathrm{p}<0,05$ ). Legend: $\mathrm{nc}=$ not calculated; DEAd $=$ stomata density in the adaxial surface; $\mathrm{DEAb}=$ stomata density in the abaxial surface; DTAd = trichome density in the adaxial surface; DTAb = trichome density in the abaxial surface.

\begin{tabular}{lccccc}
\hline Espécies & Tratamentos & $\begin{array}{c}\text { DEAd } \\
\left(\mathbf{n}^{\circ} \cdot \mathbf{m m} \mathbf{~ m}^{-\mathbf{2}}\right)\end{array}$ & $\begin{array}{c}\text { DEAb } \\
\left(\mathbf{n}^{\circ} \cdot \mathbf{m m}^{-\mathbf{2}}\right)\end{array}$ & $\begin{array}{c}\text { DTAd } \\
\left(\mathbf{n}^{\circ} \cdot \mathbf{m m}^{-\mathbf{2}}\right)\end{array}$ & $\begin{array}{c}\text { DTAb } \\
\left(\mathbf{n}^{\circ} \cdot \mathbf{m m}^{-\mathbf{2}}\right)\end{array}$ \\
\hline Maytenus & Pleno sol & $\mathrm{nc}$ & $336(51,93) \mathrm{a}$ & $\mathrm{nc}$ & $\mathrm{nc}$ \\
ilicifolia & Meia-sombra & $\mathrm{nc}$ & $241(39,98) \mathrm{a}$ & $\mathrm{nc}$ & $\mathrm{nc}$ \\
& Sombra & $\mathrm{nc}$ & $172(22,97) \mathrm{b}$ & $\mathrm{nc}$ & $\mathrm{nc}$ \\
Vitex & Pleno sol & $\mathrm{nc}$ & $339(34,63) \mathrm{a}$ & $\mathrm{nc}$ & $134(27,21) \mathrm{a}$ \\
megapotamica & Meia-sombra & $\mathrm{nc}$ & $314(29,92) \mathrm{a}$ & $\mathrm{nc}$ & $93(27,21) \mathrm{b}$ \\
& Sombra & $\mathrm{nc}$ & $180(39,29) \mathrm{b}$ & $\mathrm{nc}$ & $73(9,58) \mathrm{b}$ \\
Mikania & Pleno sol & $\mathrm{nc}$ & $147(16,0) \mathrm{a}$ & $1,92(0,34) \mathrm{a}$ & $4,95(0,38) \mathrm{a}$ \\
glomerata & Meia-sombra & $\mathrm{nc}$ & $141(11,1) \mathrm{b}$ & $1,38(0,28) \mathrm{b}$ & $3,83(0,87) \mathrm{b}$ \\
& Sombra & $\mathrm{nc}$ & $123(7,8) \mathrm{c}$ & $1,44(0,35) \mathrm{b}$ & $2,98(0,5) \mathrm{c}$ \\
Bauhinia & Pleno sol & $176,1(37,7) \mathrm{a}$ & $600(124,6) \mathrm{a}$ & $\mathrm{nc}$ & $56,5(13,8) \mathrm{a}$ \\
forficata & Sombra & $111,7(26,5) \mathrm{b}$ & $357(30,8) \mathrm{b}$ & $\mathrm{nc}$ & $53,7(12,7) \mathrm{a}$ \\
\hline
\end{tabular}

Os valores médios de clorofila $a, b$, total e razão $a / b$ não variaram entre os tratamentos para $M$. glomerata e B. forficata. Os valores médios de clorofila b e clorofila total, no tratamento pleno sol, foram menores que nas outras condições de intensidade luminosa, com diferenças significativas para $V$. megapotamica, enquanto que em M. ilicifolia esses valores foram maiores no mesmo tratamento, quando comparado com os demais. 
Tabela 3. Valores médios e respectivos desvios padrões (entre parênteses) das espessuras - total e dos tecidos foliares -, e relação $\mathrm{Pp} / \mathrm{Pe}(\mathrm{n}=8)$ das quatro espécies medicinais sob três condições de luminosidade. Letras diferentes para a mesma variável (mesma coluna), por espécie, significam que os valores são estatisticamente significantes (Fisher, $\mathrm{p}<0,05$ ). Legenda: $\mathrm{nc}=$ não calculado; $\mathrm{Pp}=$ parênquima paliçádico; $\mathrm{Pe}=$ parênquima esponjoso.

Table 3. Mean values and respective standard deviation (between parenthesis) of total and leaf tissues thickness and $\mathrm{Pp} / \mathrm{Pe}$ rate $(\mathrm{n}=8)$, of four medicinal species under three light conditions. Different letters for the same variable (same column), per species, mean that the values are statistically significant (Fisher, $\mathrm{p}<0,05$ ). Legend: $\mathrm{nc}=$ not calculated; $\mathrm{Pp}=$ palisade parenchyma; $\mathrm{Pe}=$ spongy parenchyma.

\begin{tabular}{lccccc}
\hline Espécies & Tratamentos & $\begin{array}{c}\text { Espessura } \\
\mathbf{P p}(\mu \mathbf{m})\end{array}$ & $\begin{array}{c}\text { Espessura } \\
\mathbf{P e}(\mu \mathbf{m})\end{array}$ & $\begin{array}{c}\text { Relação } \\
\mathbf{P p} / \mathbf{P e}\end{array}$ & $\begin{array}{c}\text { Espessura } \\
\text { Total }(\mu \mathbf{m})\end{array}$ \\
\hline Maytenus & Pleno sol & $117,30(23,56) \mathrm{a}$ & $147,83(28,25) \mathrm{a}$ & $0,89(0,21) \mathrm{a}$ & $315(37,61) \mathrm{a}$ \\
Ilicifolia & Meia-sombra & $109,26(34,35) \mathrm{a}$ & $146,22(21,56) \mathrm{a}$ & $0,71(0,20) \mathrm{a}$ & $307(49,26) \mathrm{a}$ \\
& Sombra & $85,16(20,86) \mathrm{a}$ & $125,33(20,96) \mathrm{a}$ & $0,67(0,21) \mathrm{a}$ & $251(24,93) \mathrm{b}$ \\
Vitex & Pleno sol & $75,52(7,14) \mathrm{a}$ & $48,20(12,06) \mathrm{a}$ & $1,56(0,59) \mathrm{a}$ & $154(14,49) \mathrm{a}$ \\
megapotamica & Meia-sombra & $65,35(14,29) \mathrm{a}$ & $47,13(6,99) \mathrm{a}$ & $1,38(0,43) \mathrm{a}$ & $149(11,26) \mathrm{a}$ \\
& Sombra & $30,53(6,23) \mathrm{b}$ & $54,63(7,58) \mathrm{a}$ & $0,55(0,08) \mathrm{b}$ & $118(15,8) \mathrm{b}$ \\
Mikania & Pleno sol & $115,2(6,6) \mathrm{a}$ & $228,3(34,6) \mathrm{a}$ & $0,51(0,07) \mathrm{a}$ & $393,1(54,0) \mathrm{a}$ \\
glomerata & Meia-sombra & $109,5(7,5) \mathrm{a}$ & $206,5(32,0) \mathrm{ab}$ & $0,54(0,08) \mathrm{a}$ & $367,1(26,9) \mathrm{ab}$ \\
& Sombra & $101,7(6,1) \mathrm{b}$ & $197,9(17,1) \mathrm{b}$ & $0,52(0,06) \mathrm{a}$ & $351,4(18,9) \mathrm{b}$ \\
Bauhinia & Pleno sol & $130,1(8,1) \mathrm{a}$ & $\mathrm{nc}$ & $\mathrm{nc}$ & $96,2(8,3) \mathrm{a}$ \\
forficata & Sombra & $100,8(5,6) \mathrm{b}$ & $\mathrm{nc}$ & $\mathrm{nc}$ & $73,2(5,6) \mathrm{b}$ \\
\hline
\end{tabular}

Somente em M. ilicifolia a concentração média de clorofila $a$ variou entre os tratamentos pleno sol e meia-sombra, não diferindo do tratamento sombra. A razão $a / b$, para essa espécie, variou entre os tratamentos pleno sol e sombra, não diferindo do tratamento meia-sombra (Tabela 4).

Tabela 4. Valores médios e respectivos desvios padrões (entre parênteses) das concentrações de clorofila (Cc) das quatro espécies medicinais sob três condições de luminosidade $(\mathrm{n}=6)$. Letras diferentes para a mesma variável (mesma linha), por espécie, significam que as diferenças entre os valores são estatisticamente significativos (Fisher, $\mathrm{p}<0,05$ ).

Table 4. Mean values and respective standard deviation (between parenthesis) of chlorophyll concentrations $(\mathrm{Cc})$ of four medicinal species under three light conditions $(\mathrm{n}=6)$. Different letters for the same variable (same line), per species, mean that the differences among the values are statistically significant (Fisher, $\mathrm{p}<0,05$ ).

\begin{tabular}{lccccc}
\hline Espécies & Tratamentos & $\begin{array}{c}\mathbf{C C} \boldsymbol{a} \\
\left(\mathbf{n m o l . c m}^{-\mathbf{2}}\right)\end{array}$ & $\begin{array}{c}\mathbf{C C} \boldsymbol{b} \\
\left(\mathbf{n m o l . c m} \mathbf{- n}^{-\mathbf{2}}\right)\end{array}$ & $\begin{array}{c}\mathbf{C C} \boldsymbol{a} / \boldsymbol{b} \\
(\mathbf{n m o l . c m})\end{array}$ & $\begin{array}{c}\mathbf{C C} \text { total } \\
\left(\mathbf{n m o l . c m}^{-2}\right)\end{array}$ \\
\hline Maytenus & Pleno sol & $46,07(23,47) \mathrm{a}$ & $26,72(37,22) \mathrm{a}$ & $2,85(1,02) \mathrm{b}$ & $72,79(60,62) \mathrm{a}$ \\
ilicifolia & Meia-sombra & $26,28(7,11) \mathrm{b}$ & $8,5(1,94) \mathrm{b}$ & $3,09(0,46) \mathrm{ab}$ & $34,79(8,78) \mathrm{b}$ \\
& Sombra & $33,91(6,13) \mathrm{ab}$ & $9,59(2,29) \mathrm{b}$ & $3,58(0,27) \mathrm{a}$ & $43,42(8,33) \mathrm{b}$ \\
Vitex & Pleno sol & $21,36(5,93) \mathrm{a}$ & $10,07(2,20) \mathrm{b}$ & $2,09(0,24) \mathrm{a}$ & $31,44(8,02) \mathrm{b}$ \\
megapotamica & Meia-sombra & $26,08(2,21) \mathrm{a}$ & $15,15(1,28) \mathrm{a}$ & $1,77(0,17) \mathrm{a}$ & $41,95(2,91) \mathrm{a}$ \\
& Sombra & $27,31(4,12) \mathrm{a}$ & $13,67(1,88) \mathrm{a}$ & $2,02(0,41) \mathrm{a}$ & $40,98(7,72) \mathrm{a}$ \\
Mikania & Pleno sol & $55,6(7,1) \mathrm{a}$ & $27,9(6,2) \mathrm{a}$ & $2,07(0,51) \mathrm{a}$ & $86,5(9,6) \mathrm{a}$ \\
glomerata & Meia-sombra & $45,4(10,3) \mathrm{a}$ & $23,3(4,9) \mathrm{a}$ & $1,97(0,33) \mathrm{a}$ & $68,8(14,0) \mathrm{a}$ \\
& Sombra & $44,1(13,5) \mathrm{a}$ & $27,2(6,3) \mathrm{a}$ & $1,63(0,44) \mathrm{a}$ & $71,4(18,1) \mathrm{a}$ \\
Bauhinia & Pleno sol & $18,8(1,9) \mathrm{a}$ & $26,2(5,2) \mathrm{a}$ & $8,5(2,02) \mathrm{a}$ & $34,8(0,2) \mathrm{a}$ \\
forficata & Sombra & $19,2(1,2) \mathrm{a}$ & $37,6(6,9) \mathrm{a}$ & $13,8(2,5) \mathrm{a}$ & $51,5(9,3) \mathrm{a}$ \\
\hline
\end{tabular}




\section{DISCUSSÃO}

As quatro espécies estudadas apresentaram padrões morfológicos diferenciados em resposta à intensidade de luz. Duas das quatro espécies estudadas (M. ilicifolia e V. megapotamica), apresentaram resultados contrários aos obtidos por vários estudos sob esse enfoque (THOMPSON et al., 1992; KLICH, 2000; HANBA et al., 2002). De uma maneira geral, as folhas, por serem órgãos muito plásticos, variam em área e massa fresca e seca, quando se desenvolvem em ambientes com diferentes intensidades luminosas (MARQUES et al., 1999; KLICH, 2000; GONÇALVES et al., 2005), de acordo com a disponibilidade de recursos locais, como água e nutrientes. Apesar da diferença entre os valores médios de área foliar, a massa foliar média (fresca e seca) de B. forficata não diferiu significativamente. Provavelmente, isso ocorreu em função da maior espessura foliar média nas folhas do tratamento pleno sol e a maior área foliar média nas folhas do tratamento bosque. Essas duas características combinadas contribuíram para o incremento da massa seca em relação à área foliar em cada tratamento, porém em proporções diferentes.

A única espécie que apresentou variação entre os tratamentos, tanto para a área quanto para a massa e o conteúdo de água nas folhas, foi $M$. glomerata, com os maiores valores médios no tratamento sombra. Plantas que recebem menor quantidade de luz tendem a produzir folhas com maior superfície para captação de luz e fixação de carbono sem risco de aquecimento ou transpiração excessiva (GATES et al., 1968; BRAGG; WESTOBY, 2002). O aumento da área foliar, nessa espécie, foi inversamente proporcional ao aumento da espessura ao longo do gradiente de luz, e isso parece representar uma estratégia para a manutenção do volume foliar (SMITH et al., 1997; EVANS, 1999; ACKERLY et al., 2000). A área foliar, juntamente com a espessura, também parece influenciar especialmente a determinação do conteúdo de água nas folhas de $M$. glomerata, indicada pela razão conteúdo de água/área foliar (mg. $\mathrm{cm}^{-2}$ ), que se manteve praticamente constante nas três condições (pleno sol: 39,6; meia sombra: 38,7; sombra: 39,4; Fisher, $\mathrm{p}>0,05$ ).

No entanto, outras características morfológicas apresentaram variações significativas, indicando que as diferentes intensidades luminosas a que essas plantas foram expostas influenciaram de alguma maneira seu desenvolvimento. A relação área/massa seca foliar (AEF) foi significativamente maior no tratamento sombra para todas as espécies. Isso se deve provavelmente à maior variação da área foliar do que da massa seca, entre os tratamentos, para a maioria das espécies. Os maiores valores de AEF geralmente ocorrem em folhas de sombra, devido à expansão foliar (DIAS-FILHO, 2000; MENDES et al., 2001; GONZÁLEZ; GIANOLI, 2004), para maximizar a captura de luz, em ambientes de luz difusa.

Outra característica importante que também reflete na variação da $\mathrm{AEF}$ e, mais precisamente na quantidade de massa verde produzida, é a espessura total da lâmina, que foi significativamente menor no tratamento sombra, para todas as espécies, principalmente quando comparada com o tratamento pleno sol. Os maiores valores na espessura da lâmina representam os ajustes estruturais nas folhas para diminuir a perda de água e aumentar a taxa fotossintética por unidade de área, em função da quantidade de luz disponível (ASHTON; BERLYN, 1994; KLICH, 2000).

Com exceção de $M$. ilicifolia, nas demais espécies o parênquima paliçádico apresentou maiores espessuras no tratamento pleno sol quando comparado com o tratamento sombra, devido ao maior número de camadas, do maior comprimento das células, ou à combinação desses dois fatores (STRAUSSDEBENEDETTI; BERLYN, 1994; MARQUES et al., 1999; MARQUES et al., 2000; MENDES et al., 2001). Tais padrões resultam das divisões celulares periclinais, que ocorrem como consequência do reconhecimento do sinal de luz que controla o sentido da divisão celular (YANO; TERASHIMA, 2004). Segundo Vogelmann et al. (1996), o formato colunar das células do parênquima paliçádico facilita a penetração da luz canalizada para o interior do mesofilo e os ajustes na espessura e na disposição das células do parênquima paliçádico proporcionam maior eficiência na distribuição da luz no interior da folha, maximizando a absorção e a fixação de carbono (SMITH et al., 1997).

A espessura do parênquima esponjoso variou significativamente apenas em M. glomerata, entre o tratamento pleno sol e sombra, sendo maior no tratamento pleno sol. Era esperado que as espessuras desse tecido fossem maiores nos tratamentos sombra e meia-sombra, uma vez que as células do parênquima esponjoso, com formato irregular, são mais eficientes na captação da luz difusa (VOGELMANN et al., 1996), predominante nesses tratamentos. Porém, a relação parênquima paliçádico/esponjoso em M. glomerata e M. ilicifolia se manteve <1, em todos os tratamentos, o que 
demonstra que o incremento dos tecidos clorofilianos foi proporcional entre eles. A razão parênquima paliçádico/esponjoso <1 é vantajosa em ambientes com menor intensidade luminosa, como um mecanismo de otimização de captura de luz (VOGELMANN et al., 1996; TAIZ; ZEIGER, 2004). Em ambientes com mais luz disponível, espera-se encontrar uma relação inversa, como observado em $V$. megapotamica nos tratamentos pleno sol e meia-sombra.

A maior densidade estomática foi encontrada nas folhas do tratamento pleno sol para todas as espécies, como observado em vários outros estudos (MARQUES et al., 1999; MILANEZE-GUTIERRE et al., 2003). Folhas sujeitas a maiores intensidades luminosas e, consequentemente, maiores temperaturas e menor umidade relativa do ar, possuem maior densidade de estômatos por unidade de área para reduzir o tempo de abertura dos estômatos durante a captura de $\mathrm{CO}_{2}$ e minimizar as perdas de água por transpiração (ASHTON; BERLYN, 1994; MARQUES et al., 1999). Com exceção de B. forficata, as espécies, em todos os tratamentos, apresentaram folhas hipoestomáticas, assim como a maioria das eudicotiledôneas de locais sombreados e úmidos (PYYKKÖ, 1979), como a Floresta Ombrófila Mista.

B. forficata possui folhas anfiestomáticas, porém a densidade estomática, por unidade de área, é menor na face adaxial do que na face abaxial. O anfiestomatismo em B. forficata se deve ao mesofilo homogêneo, formado apenas por parênquima paliçádico, que possui poucos espaços intercelulares. Estômatos nas duas faces favorecem as trocas gasosas (GIVNISH, 1988; THOMPSON et al., 1992) nas camadas mais internas do mesofilo, diminuindo a distância a ser percorrida pelos gases, pela adição de uma nova camada limítrofe de gases na face adaxial (MOTT et al., 1982; THOMPSON et al., 1992; LEE et al., 2000).

Semelhante à densidade de estômatos, o número de tricomas por unidade de área também foi maior no tratamento pleno sol para V. megapotamica e $M$. glomerata, sendo que esta última foi a única espécie com tricomas em ambas as faces da epiderme, sendo que na face adaxial ocorreram mais tricomas por unidade de área do que na face abaxial. Tricomas mais densos atenuam a temperatura, assim como refletem os raios solares mais intensos no tratamento pleno sol (TATTINI et al., 2000).

As espécies estudadas tiveram padrões diferenciados para as concentrações de clorofila, ao contrário do esperado. Enquanto que em M. glomerata e B. forficata, as concentrações de clorofila foram semelhantes entre os tratamentos, em $V$. megapotamica, as menores concentrações de clorofila $b$ e total ocorreram no tratamento pleno sol, e em M. ilicifolia ocorreu o inverso. Vários fatores podem ter influenciado os dados obtidos, como a estrutura dos tecidos clorofilianos, uma vez que a espessura e a organização dos tecidos componentes da lâmina são elementos importantes na concentração de clorofila (EVANS, 1999). Outro aspecto a ser considerado é que neste estudo avaliou-se a concentração de clorofila por unidade de área, que é dependente da espessura da folha. Provavelmente, concentrações de clorofila por unidade de massa podem apresentar resultados contrastantes, como evidenciado por Cao (2000) e Gonçalves et al. (2005).

Vários estudos sugerem que folhas de sombra usualmente possuem maior concentração de clorofila por unidade de área, como em $V$. megapotamica, e maior relação de clorofila b/a do que as folhas de sol (CAO, 2000; YANO; TERASHIMA, 2004), para incrementar a absorção da luz vermelha, que é limitada em ambientes sombrios, e para a manutenção do balanço energético entre os fotossistemas PSII e PSI (CAO, 2000). Os resultados aqui apresentados, apesar de inconclusivos, indicam que essas espécies, a exemplo de outras, possuem diferentes estratégias para adaptar seu aparato fotossintético às condições diferenciadas de luz (GIVNISH, 1988; HANBA et al., 2002; GONÇALVES et al., 2005).

A análise comparativa das características morfológicas entre os três tratamentos para as quatro espécies estudadas mostrou que $M$. ilicifolia, V. megapotamica e $B$. forficata apresentaram os mesmos padrões de variação, excetuando-se a área foliar (que variou em B. forficata), a espessura do parênquima paliçádico (que não variou em M. ilicifolia) e a concentração de clorofila (que foi diferente entre as três espécies). M. glomerata foi a espécie com maior plasticidade foliar, com exceção da concentração de clorofila. Um dos fatores que provavelmente influenciou as variações morfológicas encontradas é a forma de vida das espécies estudadas. M. ilicifolia, $V$. megapotamica e B. forficata possuem hábito arbustivo/arbóreo, cujos aspectos de crescimento e desenvolvimento do caule, ramos e folhas são semelhantes entre si, diferente do padrão de crescimento de $M$. glomerata, que é uma planta herbácea com hábito volúvel (trepador), o que lhe confere mais flexibilidade na distribuição das folhas e, consequentemente, nos ajustes morfológicos para a captura de luz (ROWE; SPECK, 2005). 
As diferenças morfológicas foram marcantes entre os tratamentos pleno sol e sombra, para todas as espécies. Os valores médios das características morfológicas foliares das plantas do tratamento meiasombra, para a maioria das espécies, foram intermediários ou acima dos demais tratamentos, apesar do tratamento meia-sombra, em termos de intensidade luminosa, se aproximar da condição de luz do tratamento sombra. Entretanto, a pequena diferença de intensidade luminosa entre os tratamentos pareceu ser suficiente para a manifestação da plasticidade de algumas características morfológicas foliares nas espécies estudadas, como observado em outros estudos (SANTIAGO et al., 2001; HANBA et al., 2002).

Concluindo, as características morfológicas avaliadas mostraram a plasticidade das espécies em relação aos tratamentos de luz, que, do ponto de vista ecológico, demonstra a capacidade para se desenvolverem em ambientes em condições heterogêneas de luz, como os sub-bosques de florestas ombrófilas. No entanto, para os produtores de plantas medicinais, o interesse reside no acúmulo de biomassa foliar, que define a massa verde foliar pretendida, com relevante importância para adoção de técnicas de manejo e ganhos de produtividade. Os valores médios de AEF indicam que o tratamento sombra é o que oferece maior biomassa, por unidade de área, para todas as espécies. Entretanto, estudos adicionais de quantificação de princípio ativo, taxas de crescimento da planta, respostas sazonais e condições nutricionais diferenciadas nos diferentes tratamentos de luz podem complementar as informações para adoção de métodos de cultivo mais eficientes dessas espécies medicinais.

\section{AGRADECIMENTOS}

À Fundação Araucária, pelo financiamento do projeto; à Capes, pela concessão de bolsa a Ana Cristina Atala Alves e Ari Espíndola Júnior.

\section{REFERÊNCIAS}

ACKERLY, D. D.; DUDLEY S. A.; SULTAN, S. E.; SCHMITT, J.; COLEMAN, J. S.; LINDER, C. R.; SANDQUIST, D. R.; GEBER, M. A.; EVANS, A. S.; DAWSON, T. E.; LECHOWICZ, M. J. The evolution of plant ecophysiological traits: recent advances and future directions. BioScience, Washington, DC, v. 50, p. 979-995, 2000.

ASHTON, P. M. S.; BERLYN, G. P. A comparison of leaf physiology and anatomy of Quercus (Section Erythrobalanus-Fagaceae) species in different light environments. American Journal of Botany, Columbus, v. 81, n. 5, p. 589-597, 1994.

BONGERS, F.; POPMA, J. Leaf characteristics of the tropical rain forest flora of Los Tuxtlas, Mexico. Botanical Gazette, Chicago, v. 151, n. 3, p. 354-365, 1990.

BRAGG, J. G.; WESTOBY, M. Leaf size and foraging for light in a sclerophyll woodland. Functional Ecology, Oxford, v. 16, p. 633-639, 2002.

CAO, K. F. Leaf anatomy and chlorophyll content of 12 woody species in contrasting light conditions in a Bornean heath forest. Canadian Journal of Botany, Ottawa, v. 78, p. 1245-1253, 2000.

CELEGHINI, R. M. S.; VILEGAS, J. H. Y.; LANÇAS, F. M. Extraction and quantitative HPLC analysis of coumarin in hydroalcoholic extracts of Mikania glomerata Spreng. ("guaco") leaves. Journal of the Brazilian Chemical Society, São Paulo, v. 12, p. 706-709, 2001.

CORRÊA, J. R.; MING, L. C.; SCHEFFER, M. C. Cultivo de plantas medicinais condimentares e aromáticas. Curitiba: Emater - PR. 1991. 162 p.

DEDECEK, R. A.; RODIGHERI, H. R. Sistemas de preparo do solo em cultivos anuais intercalados em erva-mate. Boletim de Pesquisa, Colombo, v. 38, p. 77-88, 1999.

DIAS-FILHO, M. B. Growth and biomass allocation of the $\mathrm{C}_{4}$ grasses Brachiaria brizantha and $B$. humidicola under shade. Pesquisa Agropecuária Brasileira, Brasília, DF, v. 35, n. 12, p. 2335-234, 2000. 
EVANS, J. R. Leaf anatomy enables more equal access to light and $\mathrm{CO}_{2}$ between chloroplasts. New Phytologist, Cambridge, v. 143, p. 93-104, 1999.

FEDER, N.; O'BRIEN, T. P. Plant microtechnique: some principles and new methods. American Journal of Botany, Columbus, v. 55, n. 1, p. 123-124, 1968.

GATES, D. M.; ALDERFER, R.; TAYLOR, E. Leaf temperatures of desert plants. Science, Washington, DC,v. 159, p. 994-995, 1968.

GIVNISH, T. J. Adaptation to sun and shade: a whole-plant perspective. Australian Journal of Plant Physiology, Melbourne, v. 15, p. 63-92, 1988.

GONÇALVES, J. F. de C.; VIEIRA, G.; MARRENCO, R. A.; FERRAZ, J. B.; JUNIOR, U. M. dos S.; BARROS, F. C. Nutritional status and specific leaf area of mahogany and tonka beans under two light environments. Acta Amazonica, Manaus, v. 35, n. 1, p. 23-27, 2005.

GONZÁLEZ, A. V.; GIANOLI, E. Morphological plasticity in response to shading in three Convolvulus species of different ecological breadth. Acta Oecologica, Paris, v. 26, p. 185-190, 2004.

HANBA, Y. T.; KOGAMI, H.; TERASHIMA, I. The effect of growth irradiance on leaf anatomy and photosynthesis in Acer species differing in light demand. Plant Cell and Environment, Kyoto, v. 25, p. 1021-1030, 2002.

KLICH, M. G. Leaf variations in Elaeagnus angustifolia related to environmental heterogeneity. Environmental and Experimental Botany, Elmsford, v. 44, p. 171-183, 2000.

KRAUS, J. E.; ARDUIN, M. Manual básico de métodos em morfologia vegetal. Rio de Janeiro: Ed. Universidade Federal Rural do Rio de Janeiro, 1997. 198 p.

LEE, D. W.; OBERBAUER, S. F.; JOHNSON, P.; KRISHNAPILAY, B.; MANSOR, M.; MOHAMAD, H.; YAP, S. K. Effects of irradiance and spectral quality on leaf structure and function in seedlings of two southeast Asian Hopea (Dipterocarpaceae) species. American Journal of Botany, Columbus, v. 87, p. 447-455, 2000.

LORENZI, H. Árvores brasileiras: manual de identificação e cultivo de plantas arbóreas nativas do Brasil. 2. ed. Nova Odessa: Plantarum, 1998. v. 2.

MAACK, R. Geografia física do Estado do Paraná. Curitiba: Imprensa Oficial do Paraná. 1981. 442 p.

MARQUES, A. R.; GARCIA, Q. S.; FERNANDES, G. W. Effects of sun and shade on leaf structure and sclerophylly of Sebastiana myrtilloides (Euphorbiaceae) from Serra do Cipó, Minas Gerais, Brazil. Boletim Botânico da Universidade de São Paulo, [São Paulo], v. 18, p. 21-27, 1999.

MARQUES, A. R.; GARCIA, Q. S.; REZENDE, J. L. P.; FERNANDES, G. W. Variations in leaf characteristics of two Miconia in the Brazilian cerrado under different light intensities. Tropical Ecology, Varanasi, v. 41, n. 1, p. 47-60, 2000.

MENDES, M. M.; GAZARINI, L. C.; RODRIGUES, M. L. Acclimation of Myrtus communis to contrasting Mediterranean light environments - effects on structure and chemical composition of foliage and plant water relations. Environmental and Experimental Botany, Elmsford, v. 45, p. 165-178, 2001.

MILANEZE-GUTIERRE, M. A.; MELLO, J. C. P.; DELAPORTE, R. H. Efeitos da intensidade luminosa sobre a morfo-anatomia foliar de Bouchea fluminensis (Vell.) Mold. (Verbenaceae) e sua importância no controle de qualidade da droga vegetal. Revista Brasileira de Farmacognosia, São Paulo, v. 13, n. 1, p. 23-33, 2003.

MOTT, K. A.; GIBSON, A. C.; O'LEARY, J. W. The adaptive significance of amphistomatic leaves. Plant Cell and Environment, Kyoto, v. 5, p. 455-460, 1982.

PIO CORRÊA, M. Dicionário de plantas úteis do Brasil e das exóticas cultivadas. Rio de Janeiro: Imprensa Nacional, 1984. 518 p. 
POORTER. L. 1999. Growth responses of 15 rain-forest tree species to a light gradient; the relative importance of morphological and physiological traits. Functional Ecology, Oxford, v. 13, p. 396-410, 1999.

PORRA, R. J.; THOMPSON, W. A.; KRIEDMANN, P. E. Determination of accurate extinction coefficients and simultaneous equations for assaying chlorophylls a and $\mathrm{b}$ extracted with four different solvents: verification of the concentration of chlorophyll standards by atomic absorption spectroscopy. Biochimica et Biophysica Acta, Amsterdam, v. 975, p. 384-394, 1989.

PYYKKÖ, M. Morphology and anatomy of leaves from some woody plants in a humid tropical forest of Venezuelan Guayana. Acta Botanica Fennica, Helsink, v. 112, p. 1-41, 1979.

RITTER, M. R.; WAECHTER, J. L. Biogeografia do gênero Mikania Willd. (Asteraceae) no Rio Grande do Sul, Brasil. Acta Botanica Brasilica, Porto Alegre, v. 18, n. 3, p. 643-652, 2004.

ROWE, N.; SPECK, T. Plant growth forms: an ecological and evolutionary perspective. New Phytologist, Cambridge, v. 166, p. 61-72, 2005.

SANTIAGO, E. J. A. de; PINTO, J. E. B. P.; CASTRO, E. M. de; LAMEIRA, O. A.; CONCEIÇÃO, H. E. O. da; GAVILANES, M. L. Aspectos da anatomia foliar da pimenta-longa (Piper hispidinervium C. DC.) sob diferentes condições de luminosidade. Ciência e Agrotecnologia, Lavras, v. 25, n. 5, p. 10351042, 2001.

SILVA, K. L.; CECHINEL FILHO, V. Plants of the genus Bauhinia: chemical composition and pharmacological potential. Química Nova, São Paulo, v. 25, p. 449-454, 2002.

SMITH, W. K.; VOGELMANN, T. C.; DELUCIA, E. H.; BELL, D. T.; SHEPHERD, K. A. Leaf form and Photosynthesis: do leaf structure and orientation interact to regulate internal light and carbon dioxide? Bioscience, Washington, DC, v. 47, n. 11, p. 785-793, 1997.

STRAUSS-DEBENEDETTI, S.; BERLYN, G. P. Leaf anatomical responses to light in five tropical Moraceae of different successional status. American Journal of Botany, Columbus, v. 81, n. 12, p. 1582-1591, 1994.

TAIZ, L.; ZEIGER, E. Fisiologia Vegetal. 3. ed. Porto Alegre: Artmed. 2004. 719 p.

TATTINI, M.; GRAVANO, E.; PINELLI, P.; MULINACCI, N.; ROMANI, A. Flavonoids accumulate in leaves and glandular trichomes of Phillyrea latifolia exposed to excess solar radiation. New Phytologist, Cambridge, v. 148, p. 69-77, 2000.

THOMPSON, W. A.; KRIEDEMANN, P. E.; CRAIG, I. E. Photosynthetic response to light and nutrients in sun-tolerant and shade-tolerant rainforest trees. I. Growth, leaf anatomy and nutrient content. Australian Journal of Plant Physiology, Melbourne, v. 19, p. 1-18, 1992.

VOGELMANN, T. C.; NISHIO, J. N.; SMITH, W. K. Leaves and light capture: light propagation and gradients of carbon fixation within leaves. Trends in Plant Science, Oxford, v. 1, n. 2, p. 65-70, 1996.

WITKOWSKI, E. T. F.; LAMONT, B. B. Leaf Specific mass confounds leaf density and thickness. Oecologia, Berlin, v. 88, p. 486-493, 1991.

YANO, S.; TERASHIMA, I. Developmental process of sun and shade leaves in Chenopodium album L. Plant Cell and Environment, Kyoto, v. 27, p. 781-793, 2004. 